\title{
A Novel Frequency-Selective Power Combiner/Divider in Single-Layer Substrate Integrated Waveguide Technology
}

\author{
Uwe Rosenberg, Senior Member, IEEE, Mehdi Salehi, Jens Bornemann, Fellow, IEEE, and Esfandiar Mehrshahi
}

\begin{abstract}
A frequency-selective power combiner/divider in single-layer substrate integrated waveguide (SIW) technology is introduced. The basic building block consists of a planar four cavity structure with three of them operated in SIW $T_{101}$ modes and one in SIW TE T21 $_{21}$ mode. Each cavity is coupled to one port of the unit. In addition the overall configuration considers couplings of each resonator to the two adjacent ones. Due to the coupling transformation properties of the $\mathbf{T} \mathbf{E}_{201}$ mode cavity, second order $3 \mathrm{~dB}$ transfer functions are obtained from each port to two adjacent ones while the fourth port is almost isolated. The novel combiner/divider is designed for $11 \mathrm{GHz}$ and prototyped on RT/Duroid 5870 substrate. Measurements verify the design process and operation of the device.
\end{abstract}

Index Terms-Power combiner, power divider, substrate integrated waveguide (SIW), waveguide filters.

\section{INTRODUCTION}

$\mathbf{T}$ HE design and applications of multi-port power combining and dividing systems are well understood, e.g., [1], [2]. In transceiver applications, a common application of power combining involves the sum (and/or differences) of two signals which are usually combined by broadband four-port networks such as couplers or rat-race rings. Such systems typically involve filters for frequency selection and/or attenuation of other bands [3], [4].

Following the common trend for miniaturization of systems, several attempts have been made recently to combine the power combining and filtering functions in a single component by attaching resonators to individual ports. Examples include filtering hybrids in microstrip circuitry [5], [6], LTCC technology [7] and coupled dielectric resonators in a waveguide housing [8]. A substrate integrated waveguide (SIW) bandpass rat-race coupler is presented in [9]. However, the design in [9] requires two substrate layers which complicates the fabrication process

Manuscript received February 21, 2013; revised April 20, 2013; accepted May 19, 2013. Date of publication June 27, 2013; date of current version August 05,2013 . This work was supported by the Natural Science and Engineering Research Council of Canada.

U. Rosenberg is with Mician Global Engineering GbR, Bremen, Germany (e-mail: uwe.rosenberg@ieee.org).

M. Salehi is with the Department of Electrical and Computer Engineering, University of Victoria, B.C., Canada and also with Shahid Beheshti University, G.C., Tehran, Iran (e-mail: msalehi@uvic.ca).

J. Bornemann is with the Department of Electrical and Computer Engineering, University of Victoria, B.C., Canada (e-mail: j.bornemann@ieee.org).

E. Mehrshahi is with the Department of Electrical and Computer Engineering, Shahid Beheshti University, G.C., Tehran, Iran (e-mail: mehr@sbu.ac.ir).

Color versions of one or more of the figures in this paper are available online at http://ieeexplore.ieee.org.

Digital Object Identifier 10.1109/LMWC.2013.2269039

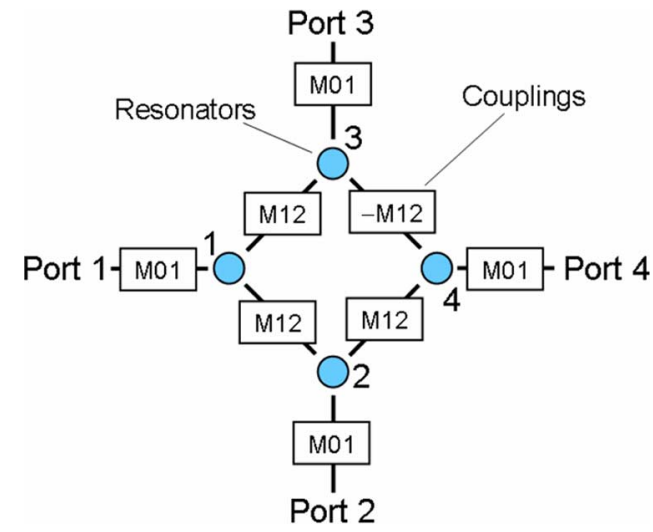

Fig. 1. Routing and coupling scheme of the frequency-selective power combiner/divider.

as the layers have to be aligned properly to realize the coupling between resonators. An all-metal waveguide design involving horizontal and vertical ports is suggested in [10]. However, neither theoretical nor experimental verification are presented.

Therefore, this letter introduces a new frequency-selective SIW power combiner/divider that can be manufactured on a single substrate layer. In principle, the component is similar to that of [10]. However, the perpendicular port is eliminated to maintain the single layer structure for a SIW realization. The measured performance of the single layer SIW implementation exhibits satisfactory coincidence with the computed results which demonstrates the feasibility of the novel design approach.

\section{Operating Principle AND Design}

Fig. 1 shows the routing scheme of the frequency-selective power combiner/divider. All four ports are coupled to their respective resonators by coupling factor $\mathrm{M}_{01}$. Inter-resonator couplings of each resonator to its adjacent ones are provided by $\mathrm{M}_{12}$. The basic structure consists of four inter-resonator couplings (cf. Fig. 1). One of these couplings has to be considered with opposite sign to achieve the coupler function, i.e., to obtain the $3 \mathrm{~dB}$ transfer functions from each port to its two adjacent ones while the opposite (fourth) port is isolated. Hence the transfer signals pass two resonators of the basic structure yielding a second order filter function. Isolation of the opposite port is achieved by the negative inter-cavity coupling so that the signals coupled from the two adjacent resonators cancel each other.

Thus theoretically, this basic structure exhibits the combination of a four port coupler like a rat-race ring or Magic Tee with a second order filter function of all transfer paths. Additional filter sections symmetrically associated at decoupled ports of 


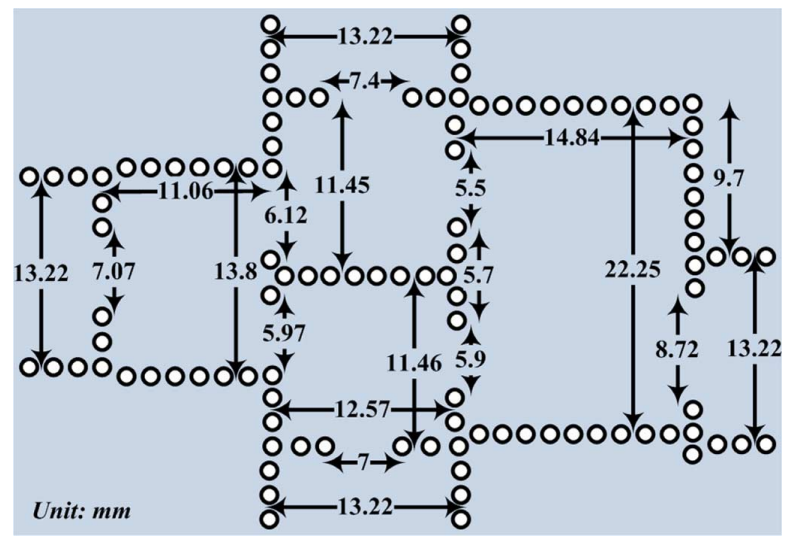

Fig. 2. Dimensions of the frequency-selective SIW power combiner/divider prototype.

the basic structure allows the realization of higher order filter functions.

The general design starts with the determination of the required filter characteristic that can be deduced from well-known filter design formulas, e.g., [11], which provide the corresponding 2-port filter $S$ parameters $S_{11 \mathrm{f}}$ and $\mathrm{S}_{21 \mathrm{f}}$ (cf. [11]). Introducing this filter $\mathrm{S}$ matrix in a classical rat-race ring or Magic Tee provides the relationship of the principle $\mathrm{S}$ matrix of the overall 4-port and the filter 2-port: $\mathrm{S}_{11}=\mathrm{S}_{22}=\mathrm{S}_{33}=\mathrm{S}_{44}=\mathrm{S}_{11 \mathrm{f}}$; $\mathrm{S}_{21}=\mathrm{S}_{31}=\mathrm{S}_{42}=-\mathrm{S}_{43}=\mathrm{S}_{21 \mathrm{f}} / \sqrt{ } 2 ; \mathrm{S}_{32}=\mathrm{S}_{41}=0$. The remaining $\mathrm{S}$ parameters follow from $[\mathrm{S}]=[\mathrm{S}]^{\mathrm{T}}$.

Thus the overall unit exhibits the same matching properties as the filter response whereas the transmission characteristics have a $3 \mathrm{~dB}$ offset according to the power splitting. This principle behavior is used to determine the objective function for the optimization of the normalized coupling coefficients (similar to [12]) and the final structure.

This approach has been applied to design a basic 4-port combiner structure with a single layer SIW implementation that exhibits a second-order Chebyshev filter characteristic. For a return loss of $20 \mathrm{~dB}$ and a bandwidth of $150 \mathrm{MHz}$ centered at $11 \mathrm{GHz}$, the normalized coupling coefficients in Fig. 1 are optimized as $\mathrm{M}_{01}=1.2247$ and $\mathrm{M}_{12}=1.1728$.

With the coupling coefficients specified, the first step is the design of an all-dielectric waveguide component using commercial software such as the $\mu$ WaveWizard. This structure is then translated to SIW technology by using equivalent waveguide widths, e.g., according to [13] and fine-optimized within CST.

Fig. 2 depicts the dimensions of the introduced single layer SIW implementation with port designations as in Fig. 1. The power combiner/divider is designed for RT/Duroid 5870 substrate with $\varepsilon_{\mathrm{r}}=2.33, \tan \delta=0.0012$, substrate thickness $\mathrm{h}=0.508 \mathrm{~mm}$, metallization thickness $\mathrm{t}=35 \mu \mathrm{m}$, and conductivity $\sigma=5.8 \times 10^{7} \mathrm{~S} / \mathrm{m}$. The via diameter is $1 \mathrm{~mm}$ with center-to-center spacing of $1.5 \mathrm{~mm}$ at all ports.

As seen in Fig. 2, the basic structure consists of three $\mathrm{TE}_{101}$-mode cavities (coupled to the left, top and bottom ports) and a $\mathrm{TE}_{201}$-mode cavity (coupled to the right port). Each cavity provides two inter-cavity couplings with its adjacent ones. The utilization of the coupling transformation properties of the $\mathrm{TE}_{201}$-mode cavity allows the realization of the

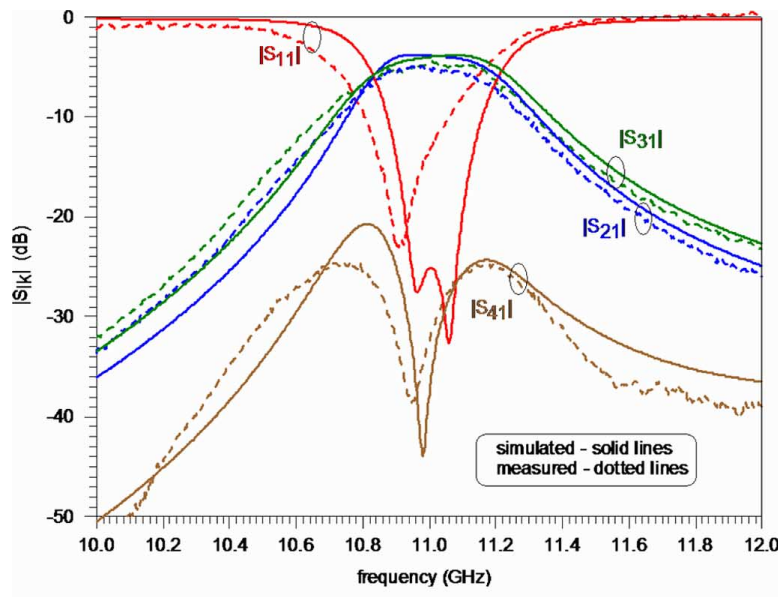

(a)

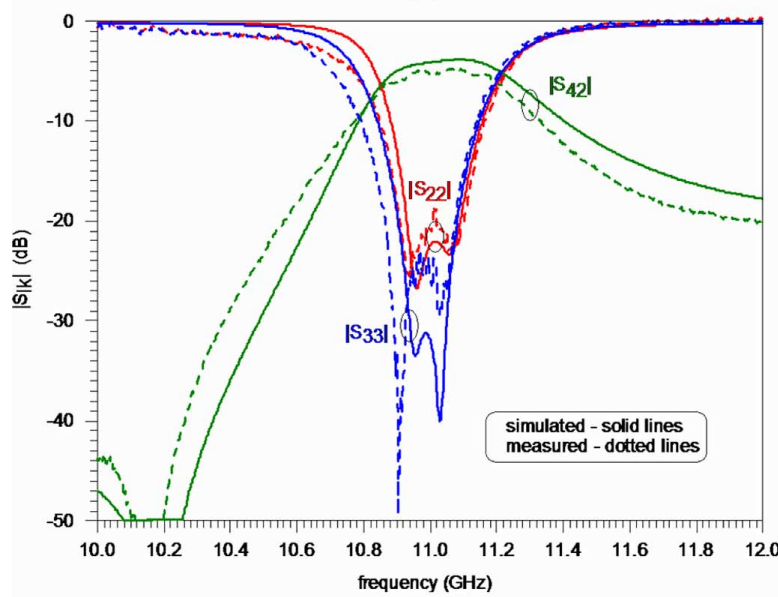

(b)

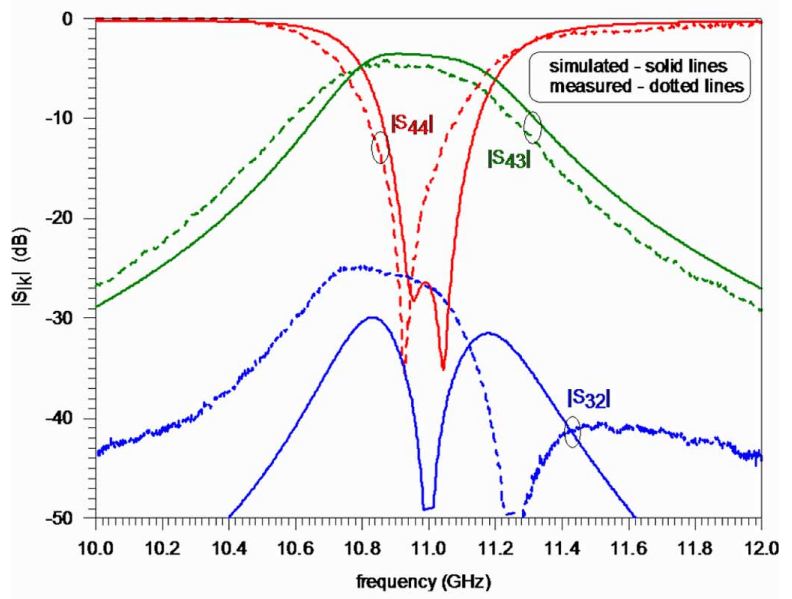

(c)

Fig. 3. Comparison between simulated and measured S parameter results: (a) $\mathrm{S}_{11}, \mathrm{~S}_{21}, \mathrm{~S}_{31}, \mathrm{~S}_{41} ;$ (b) $\mathrm{S}_{22}, \mathrm{~S}_{33}, \mathrm{~S}_{42} ;$ (c) $\mathrm{S}_{44}, \mathrm{~S}_{43}, \mathrm{~S}_{32}$ (cf. Fig. 4 for port designations).

required coupling with opposite sign-i.e., the two inter-cavity couplings are implemented at the different 'half waves' of this resonance mode (cf. Fig. 2). Note that the port coupling of the $\mathrm{TE}_{201}$-mode in a planar $\mathrm{H}$-plane structure has to be considered at one 'half wave' of the resonance mode. Due to this fact, the structure becomes completely asymmetric. For the realization, this imposes that each cavity and iris have 


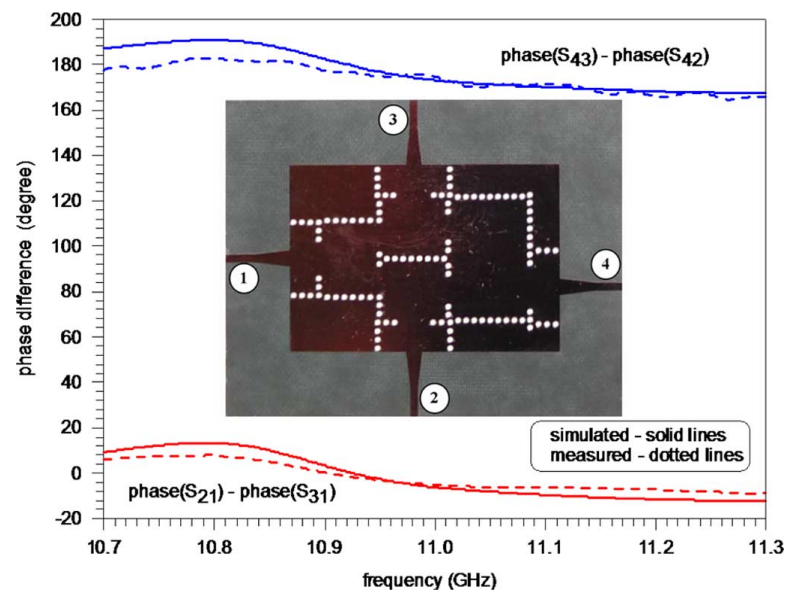

Fig. 4. Photograph of fabricated prototype and port numbering; simulated and measured phase difference between ports 2 and 3 to port 1 and port 4 .

individual dimensions to electrically accommodate the respective symmetric theoretical values. These structural differences yield inherent imperfections of this implementation, because the exact electrical values of the resonances and couplings are obtained at the center frequency. Despite these effects, it is demonstrated below that the SIW implementation of this novel design provides satisfactory performance.

\section{RESULtS}

Fig. 3 shows a comparison between simulated (CST) and measured $\mathrm{S}$ parameter magnitudes of the frequency-selective SIW power combiner/divider prototype. Note that microstrip-to-SIW transitions (cf. inset of Fig. 4) and coaxial end launchers are added for measurements. Such transitions are deembedded at the two ports that are measured while the remaining two ports are terminated with $50 \Omega$ coaxial loads.

The design and functionality of the device is well verified by measurements (Fig. 3). First, results demonstrate that despite the structural imperfections from the theoretical circuit approach, a satisfactory component design is achieved with more than $20 \mathrm{~dB}$ isolation between the respective ports. Some discrepancies between simulated and measured performances are observed with respect to the center frequency and mainly $\left|\mathrm{S}_{11}\right|$, $\left|\mathrm{S}_{32}\right|$, and $\left|\mathrm{S}_{44}\right|$. The slight shift in center frequency is mainly caused by the tolerance of \pm 0.02 of the dielectric constant of the substrate as has been verified by using $\varepsilon_{\mathrm{r}}=2.35$ in simulations (not shown here). This fact also changes some of the couplings and also moves the isolation minimum of $\left|\mathrm{S}_{32}\right|$ (top and bottom port, cf. Fig. 4) towards higher frequencies. (Note that better bandpass characteristics require a more accurate fabrication process.) However, the measured isolation is still better than $25 \mathrm{~dB}$ [Fig. 3(c)] with all other parameters performing reasonable in view of the frequency shift. The insertion losses are measured as $2 \mathrm{~dB}\left[\left|\mathrm{~S}_{21}\right|\right.$ in Fig. 3(a)], $1.5 \mathrm{~dB}$ [|S $\mathrm{S}_{31} \mid$ in Fig. 3(a)], $2 \mathrm{~dB}\left[\left|\mathrm{~S}_{42}\right|\right.$ in Fig. 3(b)] and $1.2 \mathrm{~dB}\left[\left|\mathrm{~S}_{43}\right|\right.$ in Fig. 3(c)] down from the theoretical value of $3 \mathrm{~dB}$. The simulated data, which includes dielectric and conductive losses, is about $1 \mathrm{~dB}$ better. The added insertion loss is attributed to the fact that during any measurement, only two of the four ports are deembedded. The reflections and losses caused by microstrip transitions and end launchers at the other two ports influence the measurements. This was verified by separate measurements on SIW filters.

In order to explore the phase relationships within the passbands, Fig. 4 displays comparison between simulated and measured phase differences for in-phase $\left(<\left(\mathrm{S}_{21}\right)-<\left(\mathrm{S}_{31}\right)\right)$ and out-of-phase $\left(<\left(\mathrm{S}_{43}\right)-<\left(\mathrm{S}_{42}\right)\right)$ combining ports. The agreement is reasonable, and it is observed that the measured phase difference is actually better than the simulated one. Over the designed $150 \mathrm{MHz}$ bandwidth centered at $11 \mathrm{GHz}$, the measured differences of the two ports are within -1.6 to $-6.4^{\circ}$ and 176 to $171^{\circ}$, respectively.

\section{CONCLUSION}

The single-layer frequency-selective SIW power combiner/divider represents in principle a combination of a rat-race ring with a BPF. It is a viable alternative compared to a different component that requires two separate SIW layers. Measurements performed with the SIW prototype confirm design and operation of the new single-layer SIW power combiner/divider.

Since this basic principle can be associated with arbitrary filter functions, providing very compact and low-loss implementations, it is well suited for frequency selective power combiner networks (e.g., butler matrices) as well as redundancy network circuitries that avoid extra filter equipment.

\section{REFERENCES}

[1] A. A. M. Saleh, "Planar electrically symmetric $n$-way hybrid power dividers/combiners," IEEE Trans. Microw. Theory Tech., vol. 28, pp. 555-563, Jun. 1980.

[2] K. Chang and C. Sun, "Millimeter-wave power-combining techniques," IEEE Trans. Microw. Theory Tech., vol. 31, pp. 91-107, Feb. 1983.

[3] H. Kiumarsi, A. Abdipour, M. R. Abkenari, and G. Moradi, "A Ka band balanced amplifier with six-port power divider," in Proc. 5th Eur. Microw. Integr. Circuits Conf., Paris, France, Sep. 2010, pp. 349-352.

[4] A. Pham, R. Ramachandran, J. Laskar, V. Krishnamurthy, D. Bates, W. Marcinkewicz, B. Schmanski, P. Piacente, and L. Sprinceanu, "Development of a millimeter-wave system-on-a-package utilizing MCM integration," IEEE Trans. Microw. Theory Tech., vol. 49, no. 10, pp. 1747-1749, Oct. 2001.

[5] C.-K. Lin and S.-J. Chung, "A compact filtering $180^{\circ}$ hybrid," IEEE Trans. Microw. Theory Tech., vol. 59, no. 12, pp. 3030-3036, Dec. 2011.

[6] F. Lin, Q.-X. Chu, and S. W. Wong, "Design of dual-band filtering quadrature coupler using $\lambda / 2$ and $\lambda / 4$ resonators," IEEE Microw. Wireless Compon. Lett, vol. 22, no. 11, pp. 565-567, Nov. 2012.

[7] C.-H. Wu and C. H. Chen, "Compact LTCC bandpass $180^{\circ}$ hybrid using lumped single-to-differential and single-to-common bandpass filters," in IEEE MTT-S Int. Dig., Boston, MA, Jun. 2009, pp. 1473-1476.

[8] H. Uchida, N. Yoneda, Y. Konishi, and S. Makino, "Bandpass directional couplers with electromagnetically-coupled resonators," in IEEE MTT-S Int. Dig., San Francisco, CA, May 2006, pp. 1563-1566.

[9] Y. J. Cheng and Y. Fan, "Compact substrate-integrated waveguide bandpass rat-race coupler and its microwave applications," IET Microw. Antennas Propag., vol. 6, pp. 1000-1006, Sep. 2012.

[10] U. Rosenberg and S. Amari, "New power distribution (combination) method with frequency selective properties," in Workshop Notes W14: Adv. N-Port Netw. Space Appl., Eur. Microw. Conf., Amsterdam, the Netherlands, Oct./Nov. 2012, pp. 1-34.

[11] R. J. Cameron, "Advanced coupling matrix synthesis techniques for microwave filters," IEEE Trans. Microw. Theory Tech., vol. 51, no. 1, pp. 1-10, Jan. 2003.

[12] S. Amari, U. Rosenberg, and J. Bornemann, "Adaptive synthesis and design of resonator filters with source/load-multiresonator coupling," IEEE Trans. Microw. Theory Tech, vol. 50, no. 8, pp. 1969-1978, Aug. 2002.

[13] M. Salehi and E. Mehrshahi, "A closed-form formula for dispersion characteristics of fundamental SIW mode," IEEE Microw. Wireless Comp. Lett., vol. 21, no. 1, pp. 4-6, Jan. 2011. 